\title{
EATRIS, a Vision for Translational Research in Europe
}

\author{
Regina Becker • Guus A. M. S. van Dongen
}

Received: 10 December 2010 / Accepted: 8 February 2011 /Published online: 5 May 2011

(C) Springer Science+Business Media, LLC 2011

\begin{abstract}
Tremendous progress has been made in biomedical research in genomics, proteomics and metabolomics, which has led to an increasing insight into molecular mechanisms related to diseases. Yet the output of disease prevention, diagnosis and treatment solutions remains low. It is against this background that several European countries have gathered to take new actions for improving translational research from bench to bedside. Surveys were conducted to identify major bottlenecks and assess the situation of translational research in Europe. While the importance of translational research is already recognized and national governments have increased their efforts and academia more and more directs its research towards translation, there are still hurdles to overcome. Thus, a European research infrastructure-EATRIS - will be established to integrate basic and clinical research in dedicated translational centres. These EATRIS Centres will provide broad access to high-end pre-clinical and clinical facilities, including the necessary translational expertise for researchers from academia and industry.
\end{abstract}

Keywords Translational research - Infrastructure · EATRIS · Translational centre - Molecular imaging · Biomarker

R. Becker $(\bowtie)$

EATRIS Project Management,

Helmholtz Centre for Infection Research (HZI),

Inhoffenstr. 7, 38124 Braunschweig, Germany

e-mail: eatris@helmholtz-hzi.de

G. A. M. S. van Dongen

Departments of Otolaryngology/Head and Neck Surgery and

Nuclear Medicine and PET Research,

VU University Medical Center,

Amsterdam, The Netherlands

\section{Introduction}

Biological and biomedical research has witnessed a significant acceleration of discoveries and data gathering. Analyses at molecular level, genomics, proteomics and metabolomics, as well as the growing use of highthroughput technologies, have led to an exponential increase in information about the underlying mechanisms of diseases. However, contrary to expectations of early genome research, this has not been materialized by an increase in the output of new therapies or diagnostics. Especially within academic research, there is a huge gap with respect to the process of transferring research findings from applied basic research to clinical practice. This gap has been recognized, and within the last decade, the area of translational research has gained much attention.

At the same time, the pharmaceutical industry is facing worrying challenges in seeking novel means of extracting value in the $R \& D$ process, due in part to dwindling drug pipelines and the looming patent cliff that many firms face. The current drug testing strategy of the industry involves testing early for safety and toxicity and then later testing for efficacy. This strategy often leads to vast sums of money being spent on testing, only to discover later that the drug is not efficacious. In fact, for every ten drugs that enter clinical testing, only one will make it to market. This is a contributory factor to a $R \& D$ process that ends up with a required developmental period up to 15 years and an average price tag of Euro 1.5 billion per novel drug brought to market. Furthermore, despite doubling of $R \& D$ expenditures by pharma industry, the output of new molecular entities (NME) in Europe has halved between 1989 and 2008 [1]. However, the real innovation crisis for patients and society is not the recent decline in NME, but the small percentage that provide 
distinguished clinical advantages to patients over existing medications. In 2005, all of the top ten best-selling drugs on the market were "copycats", drugs that hit precedented targets and work by the same mechanism of action as other approved drugs [2]. Thus, the pharmaceutical R\&D pipeline is in serious need of making drug development and testing more efficient and effective, as well as finding new innovative targets that meet real medical needs. Academic labs that are doing basic science and are interested in applying their discoveries to drug discovery are predestinated to find cures for diseases with high unmet medical needs by focusing on innovative targets and therapeutics.

\section{Academic Research as an Important Basis for Novel Drugs}

Most important biopharmaceutical innovations have originated from ideas and discoveries in universities and basic research centres, and the academia is more and more interested in applying their research findings to drug discovery $[3,4]$. But advancing ideas on the developmental path from basic research observations to clinical applications is often hampered within academia. Some common bottlenecks lay in the culture of biomedical research itself. There is a lack of exchange between the different disciplines, e.g. mostly a physical separation exists between clinical and basic research. Publication and citation, rather than patents, are highly valued. Nonetheless, activities of universities and public research centres in drug discoveries are increasing, despite facing many challenges. It is against this background that several European countries have gathered to take concerted new actions for improving translational research from bench to bedside. They have identified centres of excellence in translational biomedical research to create EATRIS, the European Advanced Translational Research Infrastructure in Medicine. EATRIS is one of the biomedical projects initiated by the European Strategy Forum on Research Infrastructure initially funded by the 7th Framework Programme of the European Union. FP7 lays emphasis on translating research for human health to maintain Europe's competitiveness in biomedical research and in health industry.

\section{Status of Translational Research in Europe}

To establish such a pan-European research infrastructure, it is important to have a clear view of the actual situation in translational research. Thus, EATRIS conducted a survey in which 54 academic non-profit institutions from 14 countries participated. ${ }^{1}$ Almost half of the questioned translation centres $(46 \%)$ have only been implemented since the year 2000, which mirrors the growing awareness of the importance of translational research in recent years. The centres rely mainly on national funding and thus, on the national economy, followed by industry and EU funding. Cancer is the most prominent research field of the surveyed translation centres, followed by cardiovascular diseases, neurological disorders and infectious or metabolic diseases. This probably reflects the structure of research funding and the priorities of national programmes. Medicinal product types which seem to be highly relevant are diagnostics and prognostic markers, while therapeutic approaches are slightly less frequent (cell therapies 69\%, drug development $65 \%$, biopharmaceuticals $42 \%$, biomaterials/bioengineering $35 \%$ ). The prevalence of diagnostics and prognostic markers might be due to different procedures for market approval, as the approval process is less complex and expensive.

Half of the translational centres use institutionalised collaborations to complement their translational infrastructure. The clinical facilities belonging to the translation centres vary widely in size, but most centres have access to clinics.

Besides the proximity of research and clinic, it was stated that specialisation in a specific research area (or specialisation of the research facility) together with good collaborations between the translation centre and the clinic was seen as the strongest asset of the translation centres. Despite the importance of focus, the centres pointed out that multidisciplinarity are essential for successful translational research.

\section{Developmental Stage and Output of the Translational Centres}

Considering the implementation dates of the centres and the average time until a treatment is approved, it is not surprising that the number of developmental candidates in any clinical phase is remarkably low in all centres; the same is true for commercialized patents, candidates licensed to other companies or market-approved treatments.

When analysing good laboratory practice (GLP) data in comparison to data acquired for the numbers of clinical phase II/III trials, an interesting relation could be seen. While among centres without GLP facilities only $7 \%$ have more than ten candidates in phase II/III trials, $45 \%$ of centres with facilities working under GLP conditions have more than ten candidates

\footnotetext{
${ }^{1}$ The survey has been set up as structured telephone interviews to guide the interviewees through the internet-based questionnaire and provide the possibility to clarify questions about content, structure and background of the survey.
} 
in phase II and III trials. Obviously, those centres which are more tailored to handle regulatory requirements have a higher output towards the clinic.

\section{Challenges in Translational Research}

Academia is the nucleus for the discovery of virtually all new ideas or the identification of new targets. Consequently the translational centres participating in the survey showed a strong prevalence of equipment and facilities that are needed for basic research. However, also equipment related to clinical research is abundant due to the proximity of research and clinical facilities. Yet, to advance an idea to initial testing in humans, further infrastructure or services are needed that are usually not available within academia such as synthesizing active pharmaceutical ingredients, toxicological work, safety data and regulatory requirements. The more expensive and complex the equipment, the less it is represented and the proportion of collaborations is larger. Thus large chemical libraries are established only in very few institutions, while most in an academic environment access these in a collaborational model. Especially in the area of imaging, it can be seen that very expensive facilities like cyclotrons and hot labs for tracer production according to good manufacturing practice (GMP) and advanced instruments like PET-CT ${ }^{2}$ and Ultra-highfield $\mathrm{MRI}^{3}$ are quite rare.

In most cases, academia lacks the access to facilities and technologies, services and expertise necessary to advance an idea towards pre-clinical and clinical phases. However, not only translational infrastructure is limited but also financial resources. Even the centres with a translational focus identified finance as a bottleneck in translational research. The traditional model of government support does not meet the costs, diversity and complexity of translational research.

Other topics unfamiliar to scientists are regulatory issues or intellectual property. Most interviewees judged lack of counselling and support for regulatory issues as a major roadblock in translational research. Yet so far, less than half of the translation centres employ regulatory affairs specialists at all. Many regulatory requirements affect the development process already at a very early stage. It has shown often in the past that a lack of awareness of researchers about these requirements lead to unnecessarily wasted time and money. It was expressed by the interviewees that a help-desk service for regulatory issues,

\footnotetext{
${ }^{2}$ PET (Positron Emission Tomography), CT (x-ray computed tomography)

${ }^{3}$ MRI (Magnetic Resonance Imaging)
}

which is open for academic researchers, would be desirable and could avoid such failures.

The centres emphasize the necessity of a multidisciplinarity in translational research. To take full advantage of this approach, basic researchers and clinicians must learn to understand and communicate with each other [5]. Training of clinical scientists in basic research and of basic researchers in clinical aspects is seen as a key factor for successful translational research. Especially as qualified staff is regarded as another limitation due to the current fragmentated nature of science.

\section{Needs of Biomedical Researchers}

In addition, the needs of the potential users of translational research infrastructure (including universities, research centres, research hospitals, SMEs-small and medium enterprises-from several European countries ${ }^{4}$ ) have been questioned in another survey.

In general, the results show that the needs for access to research facilities and services depend largely on the specific research activities of the research institutions, as well as on their research environment. However, they also underline the necessity to integrate basic and clinical research. Academic researchers expressed a strong need for patient-related offerings such as biobanks, access to patient cohorts and clinical phase I/II facilities - usually not available in academia. Additionally, specific pre-clinical services such as access to libraries are requested.

It is surprising that apparently many research hospitals do not yet have (sufficient) testing facilities for clinical phase I trials nor the corresponding expertise. Accordingly, they show an even higher interest in counselling in regulatory and ethical issues and first-in-man phase I clinical trials than the other research institutions.

SMEs also expressed their interest in access to patient cohorts, but also to well-equipped and GLP laboratories and clinical phase I facilities. Additionally, they would seek support for the design of clinical phase I studies. It can be seen that their interests are strongly focussed on forwarding their development but they seem to be able to cover the IPrelevant steps of the pre-clinical development already.

\section{Conclusion}

The surveys provided interesting insight into the status of translational research in Europe. While the awareness of the importance of translational research is already there and

\footnotetext{
$\overline{{ }^{4} \text { In total, } 161}$ researchers from 28 countries participated in the EATRIS survey conducted with an online questionnaire.
} 
novel centres are being built up, a lot of bottlenecks still need to be overcome and processes have to be optimised. Especially important goals which need to be achieved are:

- Provision of infrastructure and services

- Integration of basic research and clinic

- Counselling in regulatory issues and clinical phase trials

- Substantiated funding concepts

- Concepts for training and education

A European infrastructure as envisioned by EATRIS could be the ideal instrument to implement the relevant actions all over Europe and to allow a faster and more efficient translation of basic research findings into the development of innovative diagnostics and medicinal products.

\section{The Concept of EATRIS: Opening the Doors}

EATRIS is purposefully designed to help overcome the challenges faced by researchers when translating results from bench to bedside. This will be achieved by establishing a research infrastructure of translational "EATRIS Centres". EATRIS will provide access to these centres, which comprise both state-of-the-art expertise and the capital-intensive facilities residing in Europe's top translational research centres and hospitals. This access is available through a collaboration approach to academia worldwide. Additional financing needed for the research and development projects is envisaged through public and private funds.

The infrastructure will be established in existing research institutions and clinics with proven track records in translational research. To build an EATRIS Centre, leading European research institutions dedicate part of their research and development capacities to EATRIS. Clinics and research centres integrate their working procedures for translational research and development to create "virtual centres", covering the entire product development chain up to the clinic (Fig. 1).

The goal is to have all necessary disciplines (basic and clinical research) close together as a strong innovation core (Fig. 2). The EATRIS translational research infrastructure will overcome fragmentation by establishing multidisciplinary teams to accompany the projects run within EATRIS. These teams bring together from the outset all clinical, scientific, regulatory and product developmentrelated aspects needed over the course of the project. This helps to ensure that all steps and potential issues during the development process are considered from the start. Meticulous quality control and continuous advancement of facilities will ensure the necessary performance needed for translation and successful transfer of EATRIS developmental candidates to industry.

According to their core expertise, the EATRIS Translation Centres will specialise in specific disease fields and on the development of products such as diagnostics, small molecular drugs, vaccines or advanced therapy medicinal products (cell and gene therapy, tissue engineering). They are complementary in their expertise and the portfolio of research services. A central coordination and support office in EATRIS serves as an access and information point and will organise the routing of researchers to the most suitable EATRIS Centre (Fig. 3).

Within the EATRIS Centre, the researchers can find the necessary support and the facilities needed for translational research as all disciplines around the development of diagnostics and therapeutics, basic research and clinical research are being made available. Access depends on the innovation of their approach and the probability that the research findings can actually lead to added value in healthcare. An EATRIS Centre can take research findings to a first proof-of-concept in human up to early clinical phase IIa studies.

EATRIS benefits from the variety of expertise and highend technological platforms available in the different
Fig. 1 The developmental chain is the sequence of consecutive R\&D steps for product development of clinical application in diagnosis, therapy or prevention. EATRIS will cover the steps highlighted in blue and offer translational support and continuity along the entire process

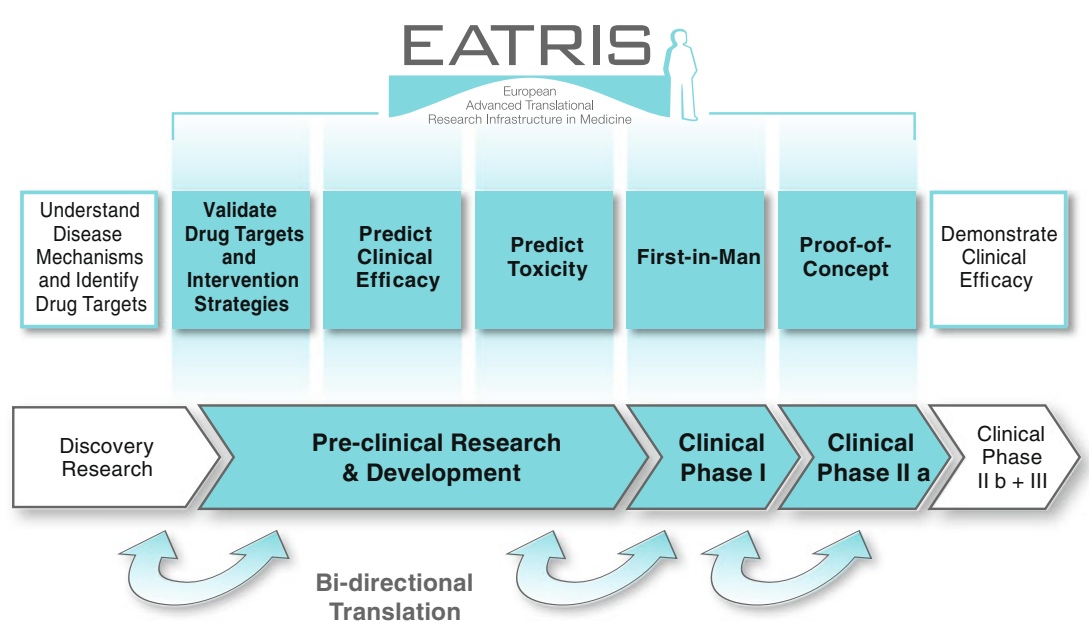




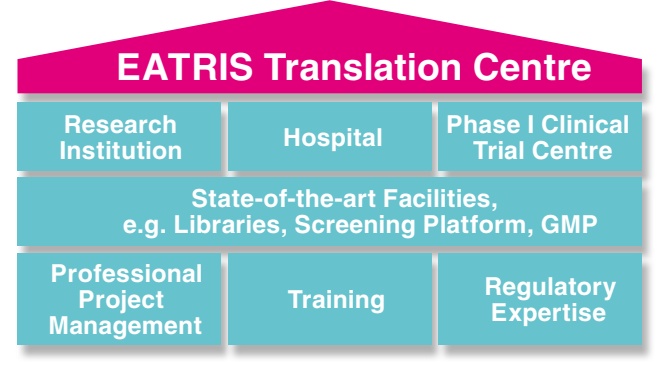

Fig. 2 In an EATRIS Centre, all necessary disciplines (basic and clinic research) are close together as a strong innovation core. They combine high-quality physical resources (so-called "bricks") and scientific expertise as well as professional project management ("brains"). This "brick and brains" infrastructure will optimally guide the scientist through the difficult process of translational medicine

participating centres. The different institutions can complement each other for a research project. In addition, common standard operating procedures and harmonised data handling will allow cross-validation of results and multi-centre studies. Innovative approaches will be achieved through the close cooperation of clinicians and scientists. The biological and technological research taking place in parallel to the translational activities lead to a cross-fertilisation and thus enable cutting edge facilities available in the development process. The technology platforms around molecular imaging and biomarker development allow in this context not only the development of new diagnostics but can be used effectively to improve the developmental process as described in the following section.

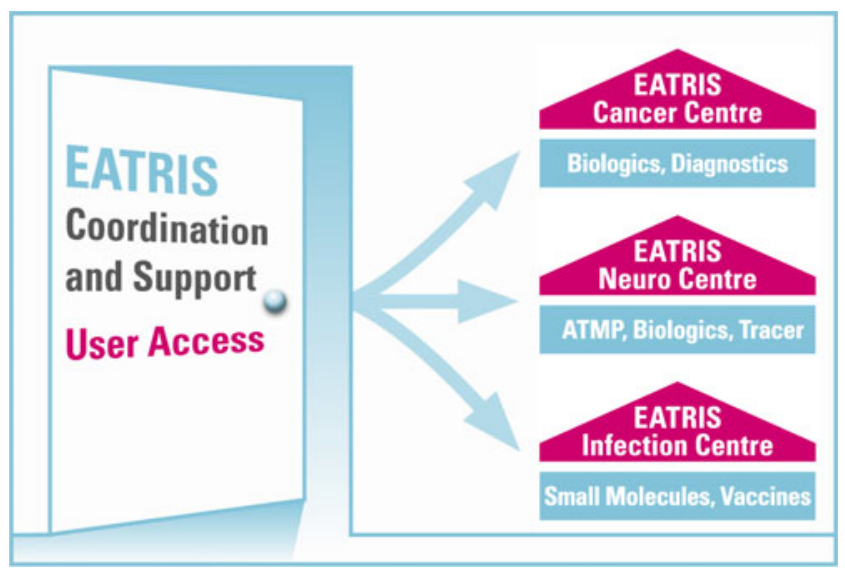

Fig. 3 The EATRIS Translation Centres will specialise in specific disease fields and on the development of products such as diagnostics, small molecule drugs, vaccines or advanced therapy medicinal products (cell and gene therapy, tissue engineering). They are complementary in their specialisation and the portfolio of research services. A central coordination office serves as an access and information point and will organise the routing of researchers to the most suitable EATRIS Centre

\section{Molecular Imaging}

Molecular imaging is a vital tool in biomedical research and drug discovery. It can be used successfully to elucidate disease mechanisms and identify new targets, contain development costs, speed up the R\&D process and increase pipeline output. It provides substantial added value in several ways.

Molecular imaging and its concomitant area of tracer development is becoming an essential tool for understanding disease mechanisms by visualising and allowing quantification of critical disease targets and molecules, be it drug candidates or diagnostic agents [6,7]. The use of molecular imaging in evaluating the efficacy of new drugs, testing early in the process (phase I/II) with small imagingenabled trials, allows the elimination of dead-end compounds before vast multi-centre trial expenditures are made. Dose-effect relations can be determined more accurately, improving the quality and efficiency of studies and reducing the risk of overdosing. The use of imaging in such dose-finding studies can allow a reduction in the number of volunteers needed in phase I by up to $80 \%$. Custom made tracers can be quickly developed in EATRIS Tracer and Molecular Imaging Centres to support researchers and companies in testing of new drugs, generally with a lead time of around 2 months or less.

Tracers can also become a unique tool in ATMP development. Tracking of cells is vital to the efficiency of the approach. EATRIS Centres can develop suitable "dyes" to mark the respective cells or molecules to make them visible in vivo.

Furthermore, molecular imaging is important to stratify patient cohorts, ensuring that the non-systemic risk of failure from sampling errors is minimised. Imaging in personalized medicine starts with disease delineation and prognostication, followed by confirmation and quantification of selective drug targeting, and finally, by early response and outcome monitoring. All of this is possible in patient cohorts displaying heterogeneous disease characteristics, to identify the cohorts with the highest potential for therapeutic benefit. One example is the use of positron emission tomography (PET) in studying the in vivo behaviour of therapeutic monoclonal antibodies (mAbs) and their interaction with critical disease targets to assess their efficacy early during treatment [7]. For expensive therapeutics like mABs to which only some patients respond, such characterisation of efficient use is vital to make them affordable to society in the long run.

Within the EATRIS Tracer and Molecular Imaging Centres, pre-clinical and clinical imaging facilities comprising PET, CT, SPECT, MRI/MRS, UltraSound, optical imaging, as well as hybrid systems will be available under the quality assurance and quality control conditions needed for translational research. The corresponding radionuclide production facilities and GMP labs, animal facilities, 
metabolite analysis and a clinical trial centre all form part of the centres. EATRIS will not only make tracers available, where needed, they can also give the necessary support to researchers in the development of their tracers and the necessary steps from laboratory to human.

\section{Biomarkers}

Biomarkers as quantifiable biological variables that characterise cellular, organ, physiological, pathological or clinical conditions play a role in various steps of drug development. Molecular diagnostics have become important means in risk analysis and identification of a disease, as well as the assessment of its severity. While many academic research institutions engage in biomarker research, few are actually validated and transferred to clinical use [8]. EATRIS wants to dedicate its efforts in Biomarker Centres to improve validation and will open its research facilities for external researchers to support them in the validation process.

In addition, EATRIS Centres engage in biomarker research in the context of drug development. Biomarkers can be used in the early discovery stages of drug development to validate the importance of the target in human diseases as well as its "druggability". They can also be used to define the interaction of a compound with a discrete target or to assess the pharmaco-kinetics of the compound-target interaction.

The most important role of biomarkers is in the field of personalised medicine. In diseases with high variability, biomarkers are employed to identify patient subgroups to allow specific therapy developments. Applied to patients indiscriminately, drugs may not be judged to be effective and thus be discarded, whereas it is may be highly effective in the subgroup. It is also well-known that some patients with certain molecular profiles react differently to their medication. This does not only affect the efficacy, but also the toxicity. Side effects up to serious adverse events can depend crucially on the molecular profile of patients. For this reason, the European Medicinal Agency (EMA) and the Federal Drug Administration increasingly ask for biomarker studies to accompany the development of new therapies for the regulatory approval.

For biomarker validation and biomarker development for patient stratification, the clinical link of EATRIS Centres is most important. It is an essential element of all EATRIS Centres that research hospitals and clinics are integrated along with well-characterised patient cohorts and biobanks. In addition, large-scale and automated screening facilities, genotyping, proteomics and metabolomics facilities typify EATRIS diagnostics centres, as well as libraries for functional screens (cDNA, shRNA, siRNA, miRNA) or antibody repositories.

\section{Therapy Development}

As mentioned above, EATRIS Centres specialise in different therapies and diseases, where they integrate several different institutions to cover the various disciplines needed and to overcome fragmentation. Each institution will have a track record in the research and development fields they cover in the EATRIS Centre. Where needed, different EATRIS Centres can complement each other for specialised activities (e.g. specific animal models such as pigs or non-human primates). Routine procedures such as formal pharmaco-kinetics and pharmaco-dynamics studies for the regulatory approval process can be bought as services from outside.

Research and development services for drug development in EATRIS range from target confirmation to finding suitable compounds and improving them to lead products in preclinical and early clinical studies. Within EATRIS, compre-

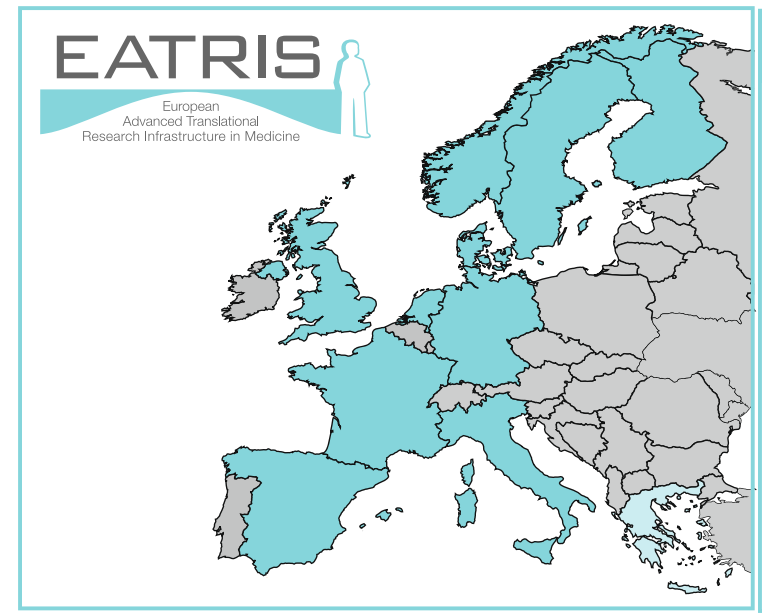

The EATRIS consortium

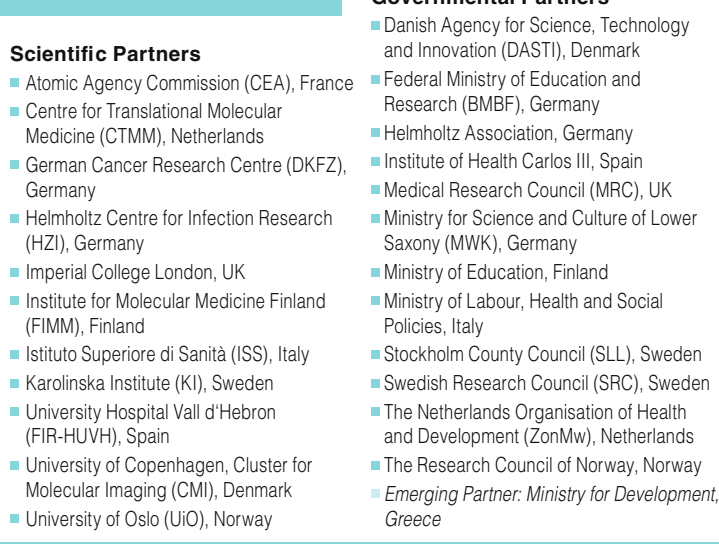

Fig. 4 Ten European countries were involved in planning the EATRIS research infrastructure (turquoise) 
hensive molecule libraries are available. It is not intended to compete with the pharmaceutical and biotech industry in this field but to bridge the gap between academia and the private sector. Better target confirmation with relevant animal models and a clear characterization of targets will improve the uptake by industry. Close interaction and open innovation collaboration with industry is key, especially in this sector. In addition, EATRIS can drive the research and development process further to a proof of concept in human in the fields of, e.g. orphan drugs where industry is reluctant to engage already in the early stages of development.

In the so-called "Advanced Therapy Medicinal Products (ATMP)" defined by the EMA to comprise cell and gene therapies as well as regenerative medicine the focus of the EATRIS support is on the specific needs in this field, namely the support of academia in setting up delivery systems and production processes which are amenable to scaling-up and cGMP conditions, as well as the subsequent production. A further service will be consultation and research about the best way to comply with regulatory requirements. Innovative new therapies in the ATMP field pose a challenge also to regulatory authorities because the current rules were set up in the context of drug development and do not transfer to the more complex conditions such as those of cell therapies [9]. EATRIS engages in these development fields and in research to find new ways for the assessment of efficacy and safety in advanced therapies. Support will be offered for all stages, from the pre-clinical studies to the clinical phase IIa trials.

\section{Outlook}

After a three-year preparatory phase (2008-2010), which had been funded by the European Commission, the EATRIS consortium (Fig. 4) is about to implement the planned pan-European translational infrastructure. A Memorandum of Understanding was signed by ministries and research councils in Finland, France, Germany, Italy, Norway, Spain and The Netherlands, who want to follow up EATRIS and create it as an independent legal entity. New candidate countries will follow.

Some of the participating countries have already launched calls to identify the institutions with the best track records in translational medicine to build an EATRIS Centres. This year, they will implement the EATRIS Centres and already start first pilot projects to demonstrate the operation of EATRIS. These activities will gradually be transferred in the offer of support on a regular basis.

EATRIS will not be a static infrastructure but will evolve following emerging new technological approaches and is intended to react to emerging medical needs. Training facilities and programs specifically dedicated to translational research will also be developed and made available to the research community. It is the aim of the participating countries to improve the translational performance of their own academic landscape and at the same time stimulate translational research in general.

\section{Summary: The Approach of EATRIS}

- Creation of a pan-European consortium for translation supporting researchers in conducting their biomedical research, from discovery to proof-of-concept in human in a lean and mean way

- Tackling fragmentation by integration of all disciplines in comprehensive virtual centres and multi-disciplinary management teams

- Engagement of specific knowledge and technology to reduce risks in development

- EATRIS will be of special importance to researchers without access to necessary translational infrastructure and expertise. Through EATRIS, they will gain access to translational centres with proven a track record.

- By offering an exchange platform to researchers in academia and industry alike, EATRIS will encourage closer collaboration.

Acknowledgement The authors acknowledge A. Ussi and B. Kuehne for the review of and suggestions for the manuscript.

\section{References}

1. EFPIA (2009). The Pharmceutical Industry in Figures, Key data, 2009 update.

2. Johnston, S. C., \& Hauser, S. L. (2006). Can industry rescue the National Institutes of Health? Annals of Neurology, 60, A11-A14.

3. Price, Waterhouse, Cooper (2009). Drug discovery and biotechnology in Germany.

4. Silber, B. M. (2010). Driving drug discovery: The fundamental role of academic labs. Science Translational Medicine, 2, 30 $\mathrm{cm} 16$.

5. Pober, J. S., Neuhauser, C. S., \& Pober, J. M. (2001). Obstacles facing translational research in academic medical centers. The FASEB Journal, 15, 2303-2313.

6. Vosjan, M. J. W. D., Perk, L. R., Visser, G. W. M., Budde, M., Jurek, P., Kiefer, G. E., et al. (2010). Conjugation and radiolabeling of monoclonal antibodies with zirconium-89 for PET imaging using the bifunctional chelate p-isothiocyanatobenzyl-desferrioxamine. Nature Protocols, 5, 739-743.

7. Van Dongen, G. A. M. S., \& Vosjan, M. J. (2010). Immuno-positron emission tomography: shedding light on clinical antibody therapy. Cancer Biotherapy \& Radiopharmaceuticals, 25(4), 375-385.

8. Braquet P (2007). Bottlenecks in Integrated biomarkers development. GDS Publishing NGPharma. http://www.ngpharma.com/ article/Bottlenecks-in-Integrated-Biomarkers-Development/. Accessed 7 November 2010.

9. Schneider, C. (2010). Challenges with advanced therapy medicinal products and how to meet them. Nature Reviews. Drug Discovery, 9, 195-201. 